\title{
Stress Singularity at the Corner Point of 3-D Surface Crack under Mode II Loading*
}

\author{
Yukitaka MURAKAMI** and Hiroshi NATSUME***
}

\begin{abstract}
The point where the front of a 3-D crack intersects a free surface is called "corner point". The ordinary elastic crack tip stress singularity of $r^{\lambda}$ is $\lambda=-0.5$. However, with regard to the stress singularity at the corner point there are several different theories. This paper concerns the detailed analysis of the stress singularity at the corner point for a through crack and a semi-circular crack under Mode II loading. According to the theories by Benthem and others, not only $K_{\text {II }}$ but also $K_{\text {III }}$ has a nonzero value at a corner point which, however, contradicts the stress-free boundary condition of the free surface. The FEM analysis of the present study based on the careful meshing and accurate determination of singularity $(\lambda)$ answers this paradox on the corner point singularity. The answer is that, although the value of $K_{I I I}$ increases as the crack front approaches the corner point, the domain of non-zero value of $K_{I I I}$ decreases to an infinitesimal value and accordingly the influence of $K_{\text {III }}$ can be ignored at the corner point. Similarly, the domain of the singular stress field with $\lambda \neq-0.5$ and $\lambda=-0.6 \sim-0.5$ also decreases as the crack front approaches the corner point. These conclusions should be considered when fracture criterion at a corner point under mixed-mode loadings is used.
\end{abstract}

Key Words : Stress Intensity Factor, Elasticity, Corner Point Singularity, Threedimensional Crack, Mixed Mode, Finite Element Method, Fracture Mechanics

\section{Introduction}

It is well known that the stress singularity of $r^{-0.5}$ for two dimensional crack under plane stress or plane strain does not hold at the corner point where the front of a $3-D$ crack intersects a free surface. According to existing analytical and numerical studies, the stress singularity $r^{\lambda}$ at the corner point is $\lambda>-0.5$ for Mode I loading and $\lambda<-0.5$ for Mode II loading ${ }^{(1)-(6)}$.

Benthem's solutions ${ }^{(1),(2)}$ of the singularity order $\lambda$ at the corner point for Poisson's ratio $\nu=0.3$ are $\lambda=$ -0.4519 for Mode I and $\lambda=-0.607$ for Mode II and III. It follows that if we calculate the stress intensity factors formally by the equation $\sqrt{2} \pi r \times$ stress, the stress intensity factor $K$ for $\nu=0.3$ along the crack front approaching a corner point becomes $K_{\mathrm{I}} \rightarrow 0$,

* Received 25th September, 2001. Japanese original: Trans. Jpn. Soc. Mech. Eng., Vol. 66, No.652, A (2000), pp. 2211-2217 (Received 19th June, 2000)

** Department of Mechanical Engineering Science, Kyushu University, 6-10-1 Hakozaki, Fukuoka 8128581, Japan. E-mail : ymura@mech.kyushu-u.ac.jp

*** Toyota Motor Ltd., Toyota, Aichi 471-0826, Japan
$K_{\mathrm{II}} \rightarrow \infty$ and $K_{\mathrm{III}} \rightarrow \infty$.

On the other hand, Su and Sun ${ }^{(7)}$ showed by the asymptotic analysis that all components of the singular stresses vanish at the corner point, though they described that the profile of the stress intensity factor in the thickness direction cannot be determined by the asymptotic analysis alone.

Finite element analysis (FEM) for a 3-D surface crack under Mode II loading was first carried out by Tohgo and Otsuka ${ }^{(8)}$ and Otsuka and Tohgo ${ }^{(9)}$. Tohgo and Otsuka ${ }^{(8)}$ analyzed the stress intensity factors for a semi-circular surface crack under Mode II loading by using $J$-integral ${ }^{(10)}$ method and showed that the value of $K_{\text {IIr }}$ increased along the crack front approaching free surface.

He and Hutchinson ${ }^{(1)}$ analyzed more in detail the Mode II and III stress intensity distribution in the vicinity of the corner point by finite element method. They also showed the increase in $K_{\text {III }}$ near the corner point. They described that in the vicinity of the corner point, the Mode III stress intensity factor does not go smoothly to zero unless Poisson's ratio is zero. Pook $^{(12)-(15)}$ and Dhondt ${ }^{(16)}$ also pointed out by 
FEM analysis the existence of a non-zero value of $K_{\text {III }}$ at corner point and, hence they supported Benthem's solutions ${ }^{(1),(2)}$. However, we should note that the corner point is on a free surface where the shear stress related to $K_{\text {III }}$ must be zero to fulfil boundary condition. He and Hutchinson's description implies that $K_{\text {III }}$ should be zero at the corner point, though they could not confirm it. It follows $K_{\mathrm{III}}=0$ from the boundary condition of free surface. Thus, the Benthem theory and the results of FEM analysis contradict the boundary condition at free surface, and yet both statements (the Benthem theory and the boundary condition) cannot be denied. How should we interpret the paradox?

Bazant and Estenssoro ${ }^{(4)}$ pointed out that a growing crack needs the condition of $\lambda=-0.5$ and in this condition the crack front intersects the free surface with a certain angle $\beta$. They showed by FEM analysis that, for $\nu=0.3, \beta=100.4 \mathrm{deg}$. for Mode I and $\beta=67.0$ deg. for Mode II and III. Considering Bazant and Estenssoro's solution, Pook suggested a partially circular surface crack with a critical intersection angle $\beta_{c}$ as a more appropriate crack model than a semicircular one.

In this paper, the characteristics of the stress singularity at corner point will be revealed in detail by systematic FEM analysis and the paradox experienced in existing studies will be solved. The solution of this problem is of substantial importance for understanding mixed mode fracture criteria.

\section{Description of the Crack Problems Analysed}

\section{1 Through crack under Mode II loading}

Figure 1 shows a rectangular plate with a through crack subjected to uniform shear stress. Considering the symmetry of the problem, FEM analysis was carried out for the hatched part of the solid in Fig. 1. Isoparametric cubic elements with 20 nodes were used. Poisson's ratio $\nu=0.3$ was assumed.

Figure 2 shows the mesh pattern. The mesh pattern in the vicinity of the crack tip where the stress intensity factor $K$ is calculated was sufficiently refined. The refinement of the mesh can be seen from the distance of the plotted data for singularity analysis which will be shown in the latter part of this paper. Figure 3 shows the boundary conditions on surfaces where, $u, v$ and $w$ are the displacements in the direction of $X, Y$ and $Z$, respectively.

\subsection{Semi-circular surface crack under Mode II loading}

Figure 4 shows a rectangular solid plate with semi-circular surface crack subjected to uniform shear stress. This model is similar to that of Otsuka and Tohgo ${ }^{(9)}$. Considering the symmetry of the prob-

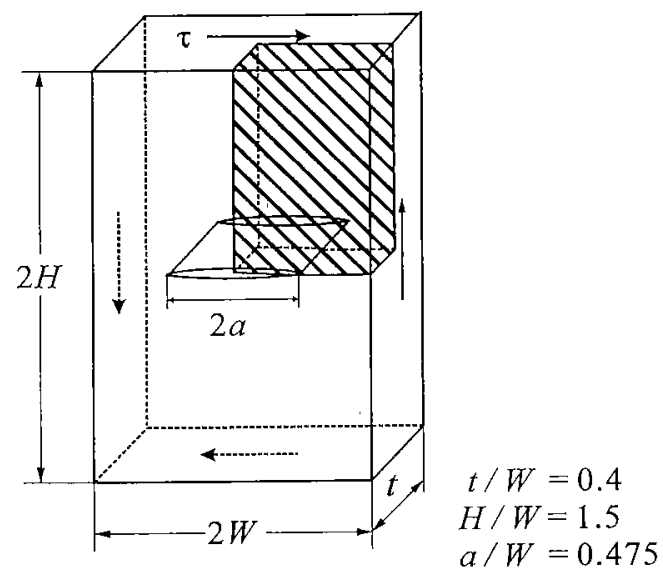

Fig. 1. Model geometry for a through crack

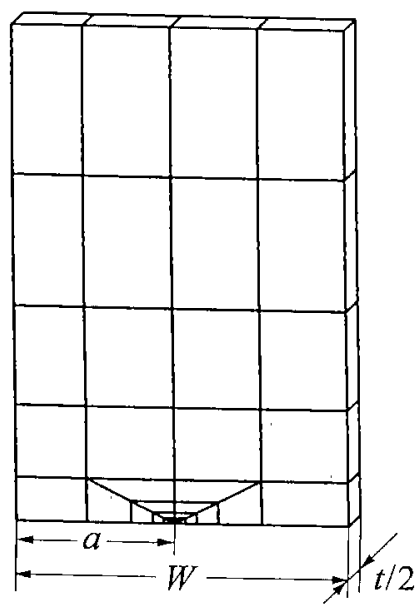

Fig. 2 Finite element mesh for a through crack

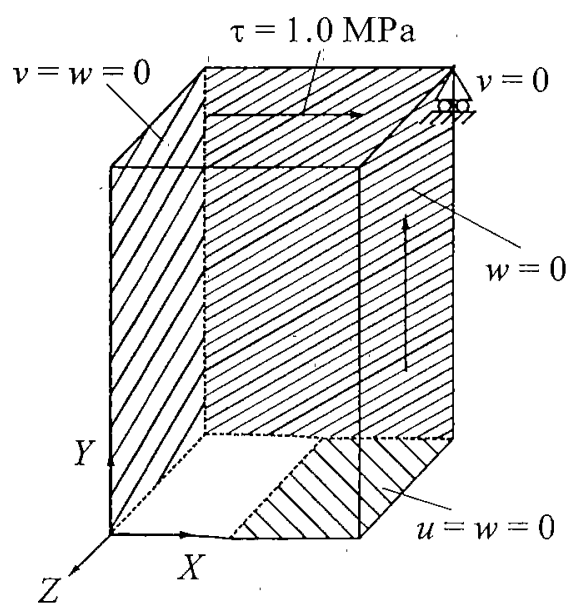

Fig. 3 Boundary conditions for a through crack

lem, FEM analysis was carried out for a quarter part of the solid. The same type of element as that of the problem of Fig. 1 was used. Poisson's ratio was also the same as that for the problem of Fig. 1. Figure 5 shows the mesh pattern. A mesh refinement similar to that of the problem of Fig. 1 was adopted. Figure 6 


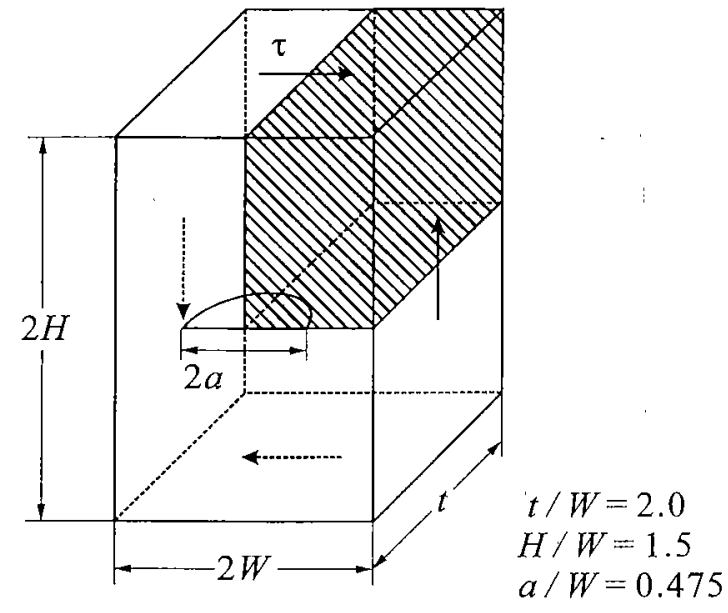

Fig. 4 Model geometry for a semi-circular crack

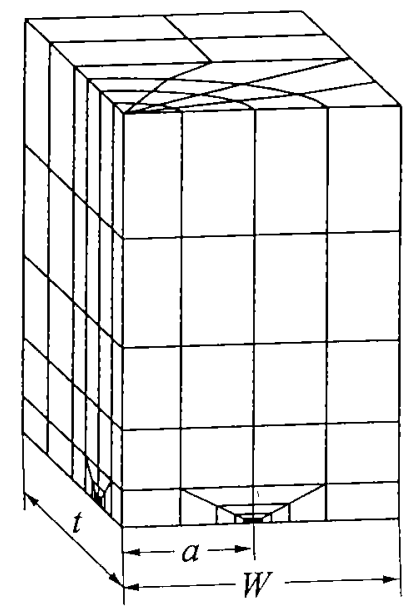

Fig. 5 Finite element mesh for a semi-circular crack

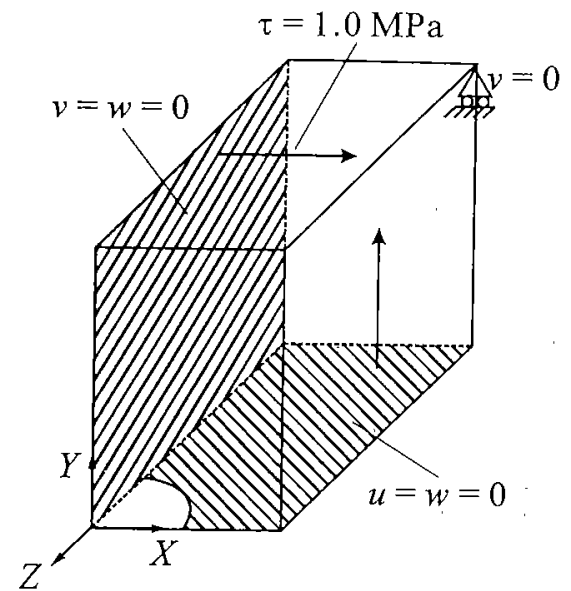

Fig. 6 Boundary conditions for a semi-circular crack

shows the boundary conditions. In the present analysis, a uniform shear stress was applied on both upper and side surfaces, whereas Otsuka applied shear stress only on upper surface.

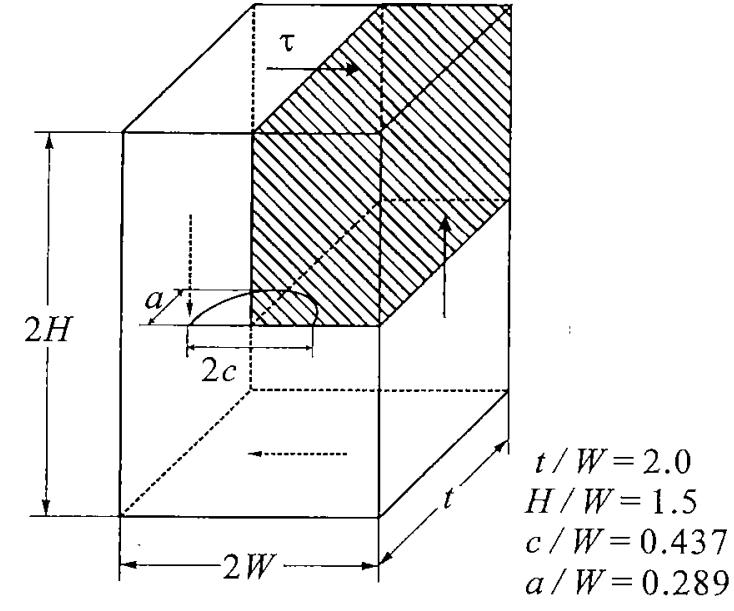

Fig. 7 Model geometry for a partially circular crack

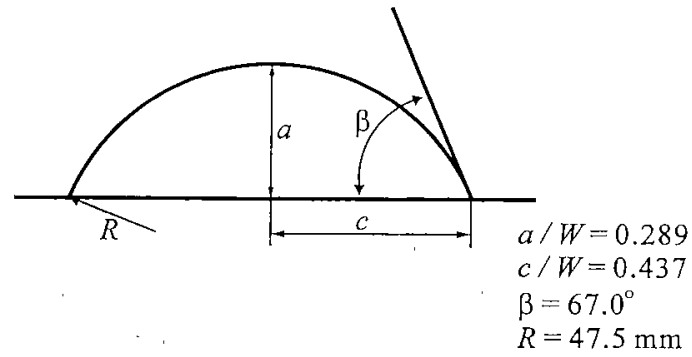

Fig. 8 Partially circular crack

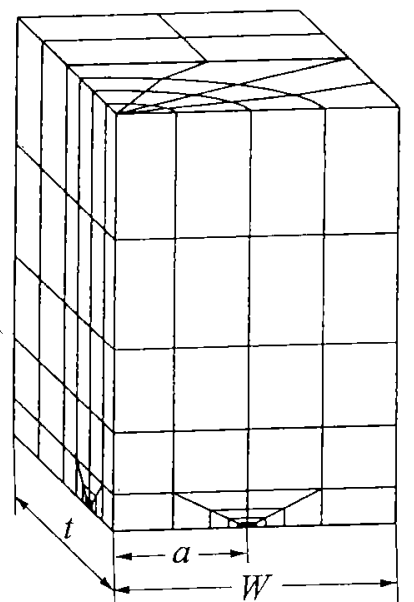

Fig. 9 Finite element mesh for a partially circular crack

\subsection{Partially circular crack under Mode II load- ing}

Figure 7 shows a rectangular solid with a partielly circular crack subjected to uniform shear stress. Except for the shape of the crack, the details of meshing (Fig. 9), loading and boundary conditions (Fig. 10), elastic constants, and FEM analysis, are the same as those of the previous problems. As shown in Fig. 8, the crack front intersects the free surface with the angle $\beta$. In the present analysis, the critical angle $\beta_{c}=67.0$, which was determined by Bazant and Estanssoro $^{(4)}$ and Pook ${ }^{(13)}$ used for FEM analysis was 


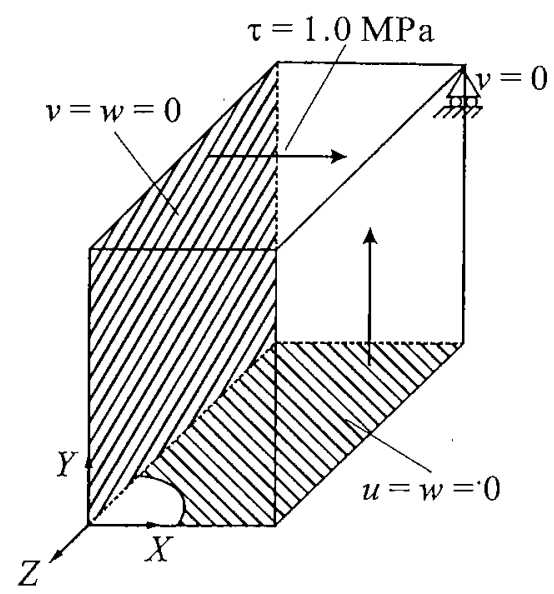

Fig. 10 Boundary conditions for a partially circular crack

considered.

\section{Results and Discussion}

\section{1 Through crack under Mode II loading}

Figures $11(\mathrm{a})$ and (b) show the stress distribution near the crack tip for the through crack problems (Fig. 1). The value of the ordinate is the shear stress $\tau_{x y}$ or $\tau_{y z}$, normalized by the remote stress $\tau$. The value of the abscissa is the distance $r$ from crack tip normalized by the half crack length $a$. The distribution of $\tau_{x y}$ corresponds to $K_{\mathrm{II}}$, and that of $\tau_{y z}$ to $K_{\mathrm{III}}$. In these figure only the values close to the free surface (corner point) are presented, $(|Z| / t$ is the nondimensional distance from the free surface and $t$ is the plate thickness).

Figure 11(a) shows that the stress distribution $\tau_{x y} / \tau$ for Mode II has the singularity of $\lambda=-0.596$ at the domain (1) which is approximately equal to Benthem's value of $\lambda=-0.607$. On the other hand, the singularity at internal points has $\lambda=-0.5$ at the domain (2) - (5) of Fig. 11(a).

Figure 11(b) shows that the stress distribution $\tau_{y z} / \tau$ at surface for Mode III is very irregular with no clear singularity and yet $\tau_{y z}$ is not zero, although $\tau_{y z}$ should be zero at the free surface because of boundary condition. The reason why $\tau_{y z}$ at the free surface is not zero is that the surface values of $\tau_{y z}$ are calculated by extrapolating the values at the internal integration points of FEM isoparametric elements. Thus, the singularity $\lambda$ for Mode III at the surface cannot be determined by the corresponding surface stress distribution. On the other hand, the stress distribution at the internal points shows the singularity of $\lambda=-0.5$ for the domain (1) - (4) of Fig. 11 (b). However, it should be noted that the finite domain having the singularity $\lambda=-0.5$ reduces to an infinitesimal value near the crack tip as the crack front approaches the

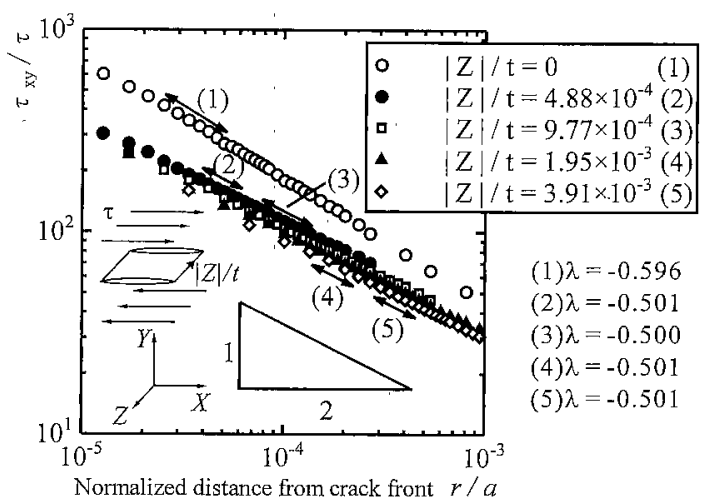

(a) Mode II stress distribution $\left(\tau_{x y}\right)$ near corner point for a through crack

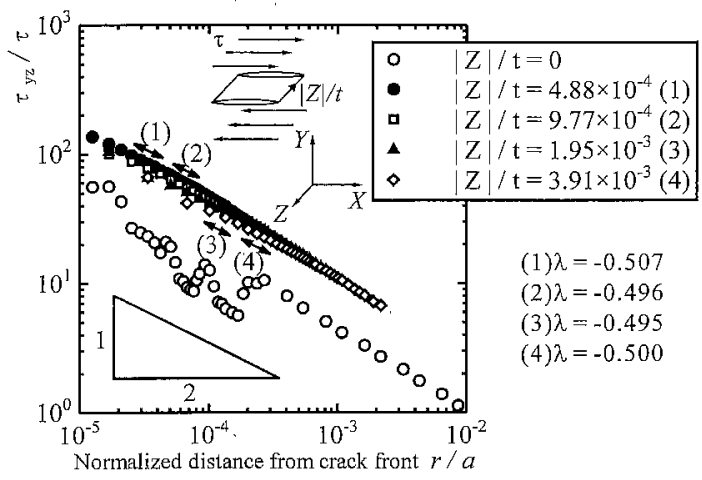

(b) Mode III stress distribution $\left(\tau_{y z}\right)$ near corner point for a through crack

Fig. 11 Stress distributions near corner point for a through crack

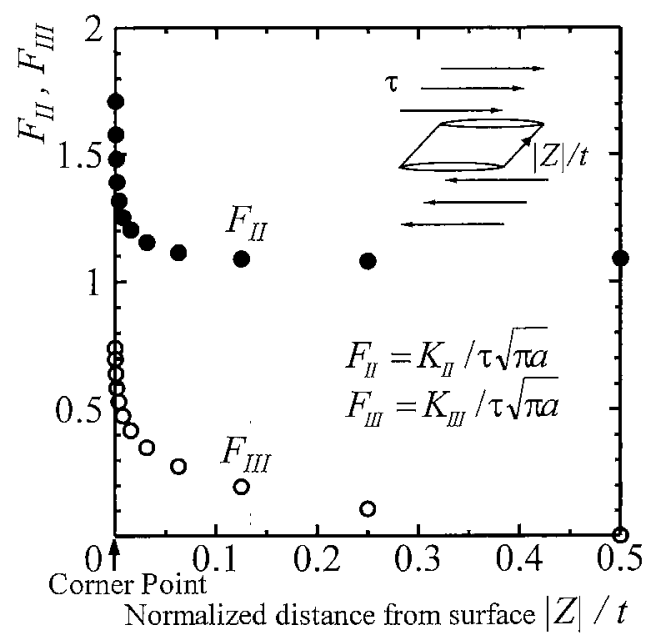

Fig. $12 K$-distribution along the crack front for a through crack

corner point.

Figure 12 shows the variation of the values of $K$ along the crack front between the midpoint of the plate and surface. The ordinate shows the correction factor $F_{\text {II }}$ and $F_{\text {III }}$ for $K_{\text {II }}$ and $K_{\text {III, }}$ and the abscissa shows the normalized distance $|Z| / t$ from the corner 


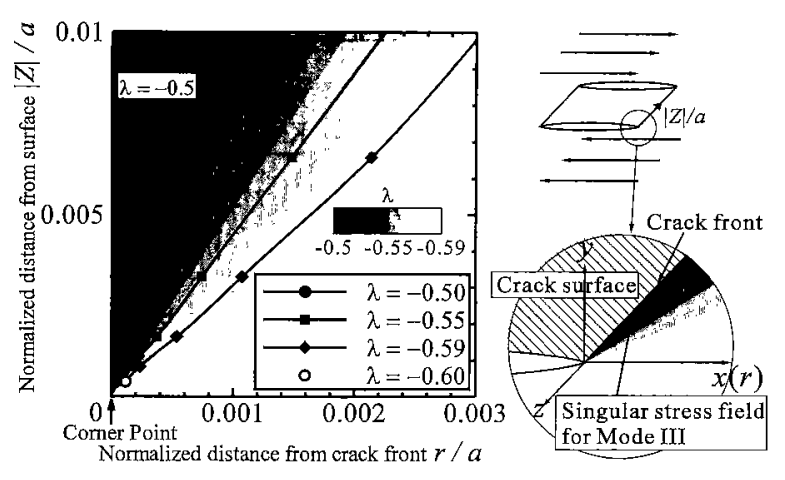

Fig. 13 Singular stress field near corner point for Mode III for a through crack

point along the crack front. The values of $K$ were calculated from the stresses which indicate the singularity of -0.5 near crack tip. As seen in the figure, $F_{\text {I }}$ has almost a constant value over the entire thickness of the plate except for the part close to the corner point where $F_{\text {II }}$ increases steeply. $F_{\text {II }}$ is zero at the center of the plate but increases toward an infinite value near the corner point. This tendency of $K_{\mathrm{III}} \rightarrow \infty$ at the corner point contradicts the boundary condition of the free surface $\left(\tau_{y z}=0\right)$ of the plate.

To make clear the aforementioned paradox, the singular stress field near the corner point was analyzed more in details. The stress distribution of $\tau_{y z} / \tau$ for Mode III in Fig. 11 ( $b$ ) shows that the value of singularity $\lambda$ varies depending on the distance from the corner point. The value of $\lambda$ is equal to -0.5 for the region ( 1$)-(4)\left(|Z| / t=4.88 \times 10^{-4} \sim 3.9 \times 10^{-3}\right)$, although $\lambda$ tends to approach -0.6 as $r / a$ increases. Moreover, the size of the domain having the singularity $\lambda=-0.5$ decreases as the crack front approaches the corner point, as shown by the stress variations from (4) to (1).

Figure 13 shows the value of the singularity from $\lambda=-0.5$ to -0.6 and the size of the domain occupied by the singularity. The ordinate shows the distance $|Z| / a$ from the surface normalized by the half crack length $a$ and the abscissa the normalized distance $r / a$ from crack front. As shown in the figure, the size of the domain of the singularity $\lambda=-0.5 \sim-0.6$ for $\tau_{y z}$ (Mode III) shrinks to zero as the crack front approaches the corner point.

Figure 14 shows the correction factors $F_{\text {II }}$ and $F_{\text {III }}$ for $K_{\mathrm{II}}$ and $K_{\mathrm{III}}$ which were calculated from the stresses at a constant distance $r / a=1.0 \times 10^{-3}$ from the crack tip along the various points of the crack front $|Z| / a$ by assuming $\sqrt{r}$ singularity of stress. As shown in Fig. 14, whereas the values of $F_{\text {II }}$ increases steeply near the corner point, the values of $F_{\text {III }}$ decreases steeply to zero near the corner point. It follows that although the value of $K_{\mathrm{III}}$ increases unboundedly

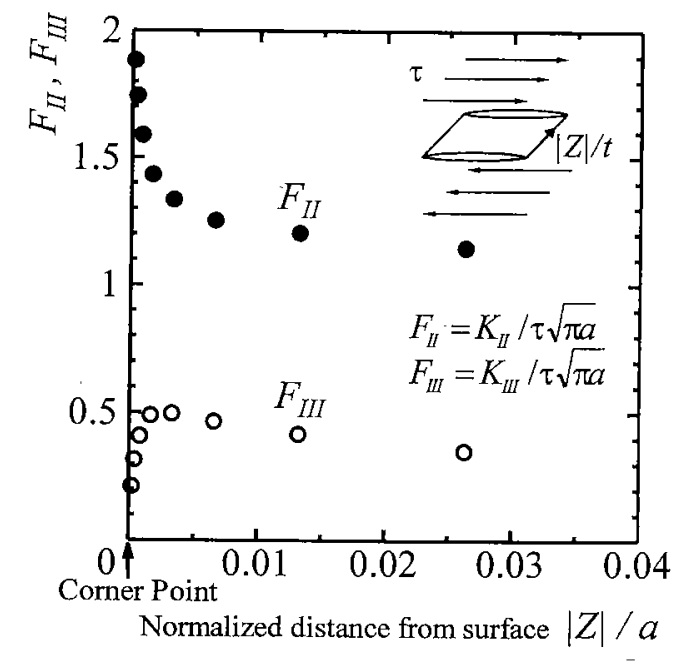

Fig. 14 Values of $K$ determined by the stress at $r / a=1.0$ $\times 10^{-3}$ for a through crack by assuming $\sqrt{r}$ singularity of stress

towards the corner point, the domain occupied by the -0.5 singularity decreases to an infinitesimal value and $K_{\text {III }}$ loses its meaning at the corner point. Figure 14 indicates that although $K_{\mathrm{III}}=0$ at the free surface (so as to fulfil the boundary condition $\tau_{y z}=0$ ), $K_{\text {III }}$ has non-zero values of approximately one third of $K_{\text {II }}$ in the domain $|Z| / a=2 \times 10^{-3}-3 \times 10^{-2}$, if we define $K_{\text {II }}$ by the stress $\tau_{y z}$ at $r / a=1.0 \times 10^{-3}$. These results indicate that the values of $K_{\mathrm{II}}$ determined by Pook $^{(12),(13)}$ and Dhondt ${ }^{(16)}$, based on coarse meshing of FEM models and extrapolation of the calculated values toward the corner point, are not correct.

3.2 Semi-circular crack under Mode II loading

Figures 15( a ) and (b) show the stress distribution near the crack tip for the semi-circular crack (Fig. 4). The value of the ordinate is the shear stress $\tau_{x y}$ or $\tau_{y z}$ normalized by the remote stress $\tau$. The value of the abscissa is the distance $r$ from crack tip normalized by the half crack length $a$. The distribution of $\tau_{x y}$ corresponds to $K_{\mathrm{II}}$ and that of $\tau_{y z}$ to $K_{\text {III. }}$ In these figures only the values close to the free surface (corner point) are presented, where $\phi$ is the counterclockwise angle from the corner point. The $x, y$ and $z$ coordinate system indicates respectively the directions principal normal, binormal and tangential to the crack front.

Figure 15(a) shows that the singularity of $\tau_{x y} / \tau$ (Mode II) is $\lambda=-0.602$ in the domain ( 1 ) at the free surface and $\lambda=-0.5$ in the domain $(2)-(4)$ at the interior close to the corner point. Figure 15 ( $b$ ) shows that there is no definite singularity of $\tau_{y z} / \tau$ (Mode III) at the free surface but the singularity in the domain (1) - (3) at the interior is approximately $\lambda=-0.5$.

Figure 16 shows the distribution of the stress intensity factors $K_{\mathrm{II}}$ and $K_{\mathrm{III}}$ in terms of the correction 


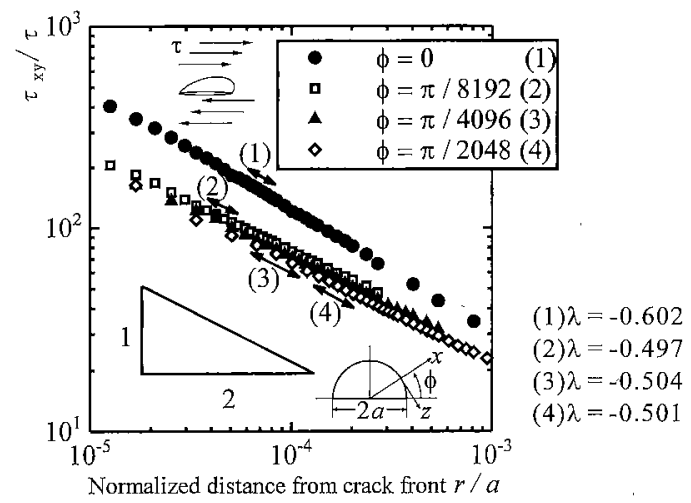

(a) Mode II stress distribution $\left(\tau_{x y}\right)$ near corner point for a semi-circular crack

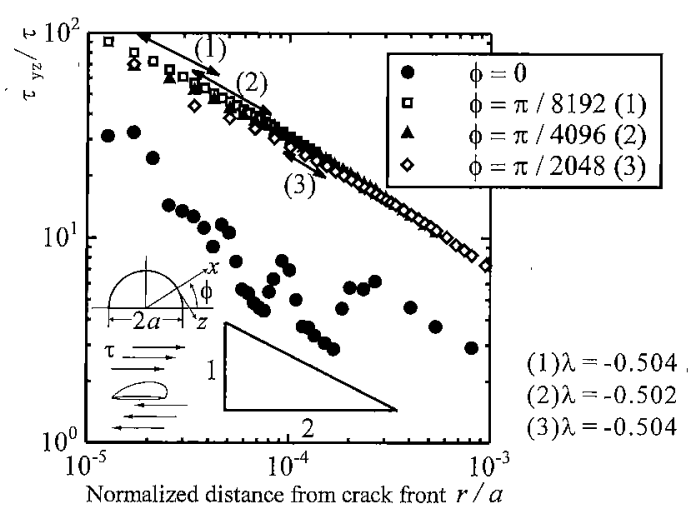

(b) Mode III stress distribution $\left(\tau_{y z}\right)$ near corner point for a semi-circular crack

Fig. 15 Stress distributions near corner point for a semi-circular crack

factors $F_{\text {II }}$ and $F_{\text {III }}$ along the crack front. The two solid curves in Fig. 16 are the exact solutions by Kassir and $\operatorname{Sih}^{(17)}$ for a penny-shaped crack in an infinite body according to which $K_{\mathrm{II}}$ at $\phi=0$ is expressed by

$$
K_{\text {II }}=\frac{4 \tau \sqrt{a}}{(2-\nu) \sqrt{\pi}}, \text { for } \phi=0
$$

According to the present FEM analysis, $F_{\mathrm{II}}=0$ at the deepest crack front $(\phi=\pi / 2)$ and increases with decreasing $\phi$, in line with Kassir and Sih's solution ${ }^{(17)}$, and finally steeply increases near the corner point.

$F_{\text {III }}$ has a maximum value at the deepest crack front $(\phi=\pi / 2)$ and decreases with decreasing $\phi$, in line with Kassir and Sih's solution, but finally steeply increases near the corner point. These distributions of stress intensity factors near the corner point are similar to those of the through-crack described in Section 3.1.

Figure 17 schematically shows the very particular stress singularity distribution near the corner point of $\tau_{y z}$ (Mode III) in detail. The ordinate of Fig. 17 is the location $|Z| / a$ of the crack front from the free surface and the abscissa is the non-dimensional distance $r / a$

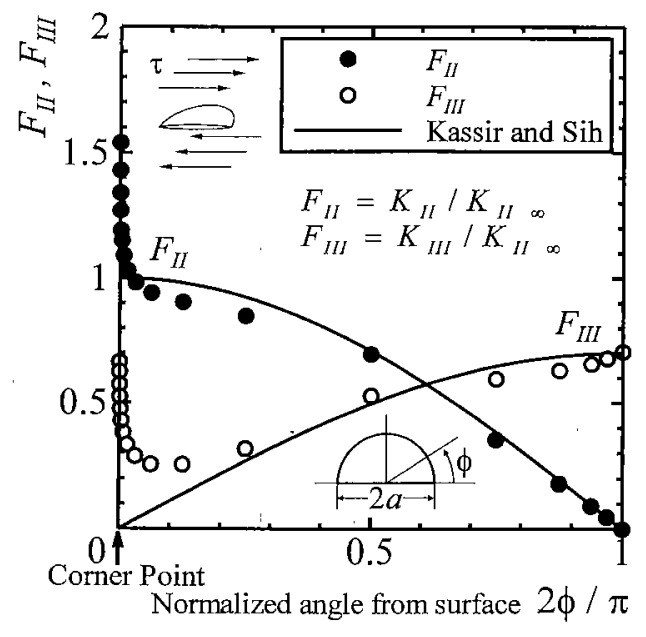

Fig. $16 K$-distribution along the crack front for a semicircular crack, $K_{\mathrm{I} \infty}=(4 \tau \sqrt{a}) /(2-\nu) \sqrt{\pi}$

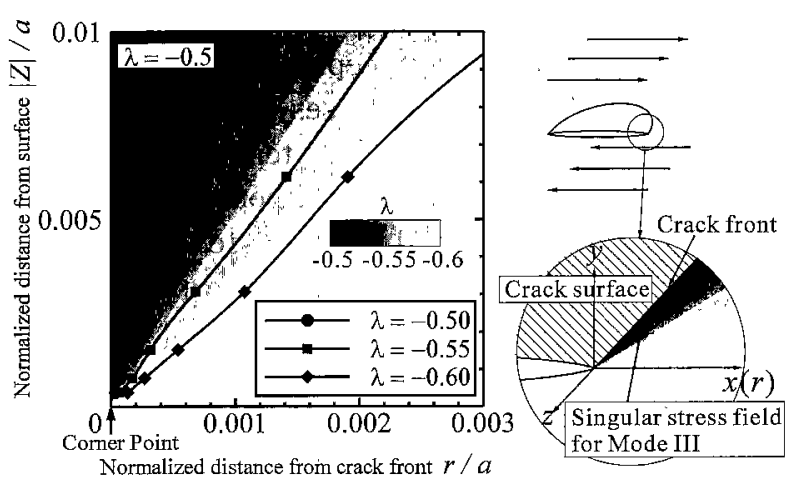

Fig. 17 Singular stress field near the corner point for Mode III for a semi-circular crack

from the crack front. As shown in Fig. 17, the domain having the singular stress distribution of $\tau_{y z}$ shrinks to zero as the crack front approaches the corner point.

Figure 18 shows the correction factors $F_{\text {II }}$ and $F_{\text {III }}$ for $K_{\text {II }}$ and $K_{\text {III }}$ which were calculated from the values of stress at a constant distance $r / a=1.0 \times 10^{-3}$ and at different location $|Z| / a$ from the free surface by assuming $\sqrt{r}$ singularity of stress. It is shown (Fig. 18) that $F_{\text {III }}$ steeply decreases to zero as the crack front approaches the free surface. This is because the domain having the $\sqrt{r}$ stress distribution for $K_{\text {III }}$ shrinks to zero and the $\sqrt{r}$ singularity at the distance $r / a=1.0 \times 10^{-3}$ from the crack front disappears as the crack front approaches the corner point. It follows that $K_{\text {III }}$ has no practical importance at the corner point similarly to the case of through crack.

These characteristics of singular stress distributions near the corner point explains why Tohgo and Otsuka ${ }^{(8)}$ obtained the finite value of $K_{\text {III }}$ at the corner point, that is, Tohgo and Otsuka used the $J$-integral method with relatively coarse meshes in their FEM analysis, and the domains shown in Fig. 17 which have 


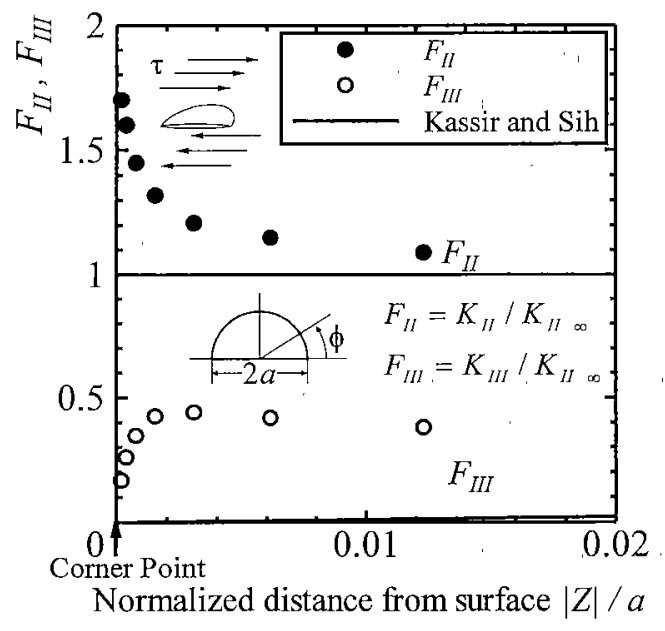

Fig. 18 Values of $K$ determined by the stress at $r / a=1.0$ $\times 10^{-3}$ for a semi-circular crack by assuming $\sqrt{r}$ singularity of stress, $K_{1[\infty}=(4 \tau \sqrt{a}) /(2-\nu) \sqrt{\pi}$

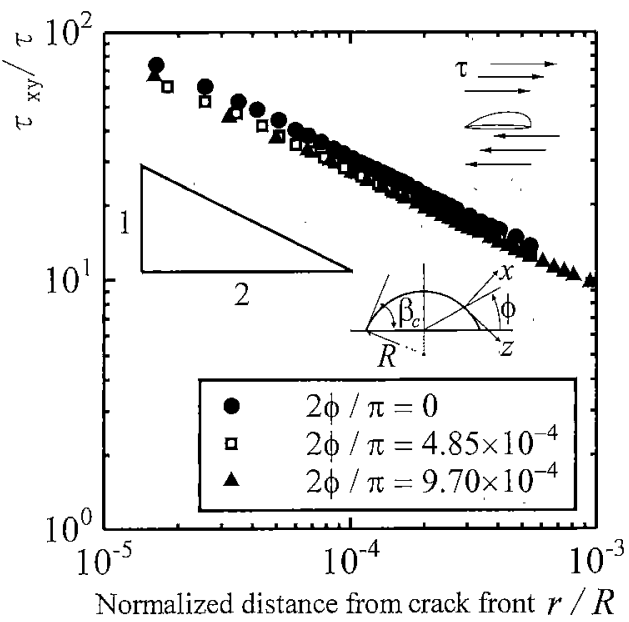

(a) Mode II stress distribution $\left(\tau_{x y}\right)$ near corner point for a partially circular crack

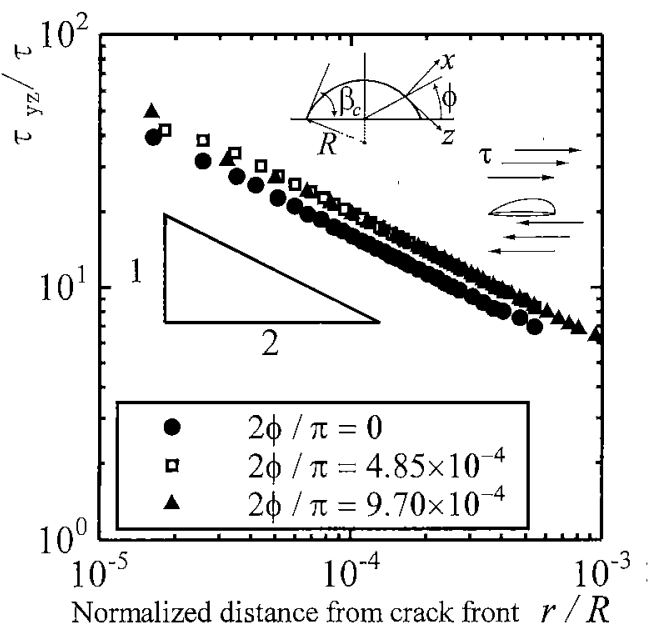

(b) Mode III stress distribution $\left(\tau_{y z}\right)$ near corner point for a partially circular crack

Fig. 19 Stress distributions near corner point for a partially circular crack

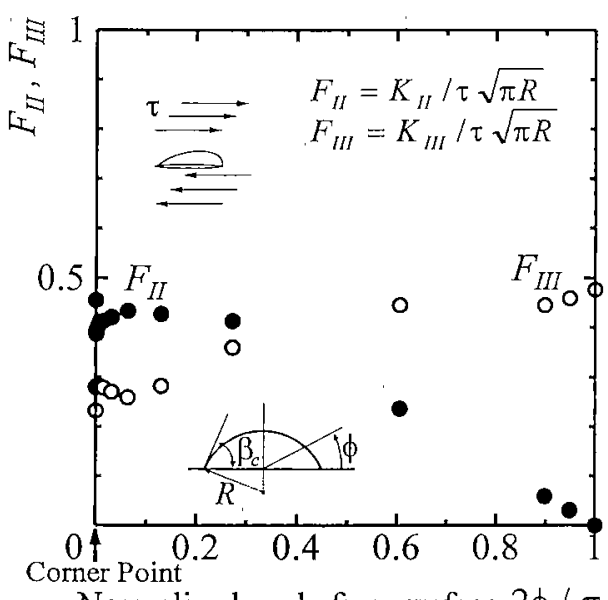

Normalized angle from surface $2 \phi / \pi$

Fig. $20 K$-distribution along the crack front for a partially circular crack, $\beta_{\mathrm{c}}=67.0^{\circ}$

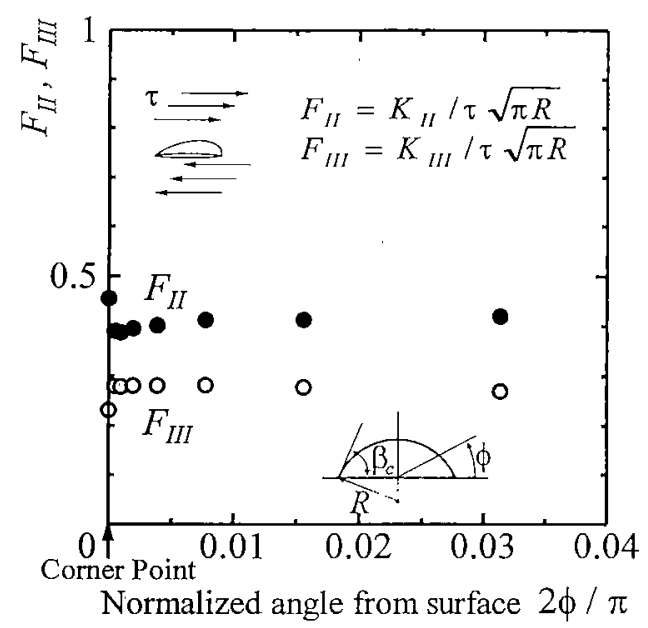

Fig. $21 K$ near corner point for a partially circular crack, $\beta_{c}=67.0^{\circ}$

the $\sqrt{r}$ singularity of Mode III near the corner point were included in the calculation of $J$-integral.

\section{3 Partially circular surface crack under Mode II loading}

Figures 19( a ) and ( b ) shows the singular stress distribution for the partially circular surface crack which has the critical intersection angle $\beta_{c}=67.0 \mathrm{deg}$. at the corner point. The ordinate is $\tau_{x y} / \tau$ and $\tau_{y z} / \tau$, and the abscissa is the normalized distance $r / a$ from the crack front. As shown in Fig. 19, both $\tau_{x y} / \tau$ and $\tau_{y z} / \tau$ have the singularity of $\lambda=-0.5$ at all points of the crack front, in agreement with the theory by Bazant et al. ${ }^{(4)}$.

Figure 20 shows the correction factors $F_{\text {II }}$ and $F_{\text {III }}$ along the crack front locations $2 \phi / \pi . \quad F_{\mathrm{II}}=0$ at the deepest point $(\phi=\pi / 2)$ and it increases with decreasing $\phi$.

Figure 21 shows the distribution of $K$ near the corner point. The definitions of the ordinate and abscissa are the same as those of Fig. 20. As indicated 


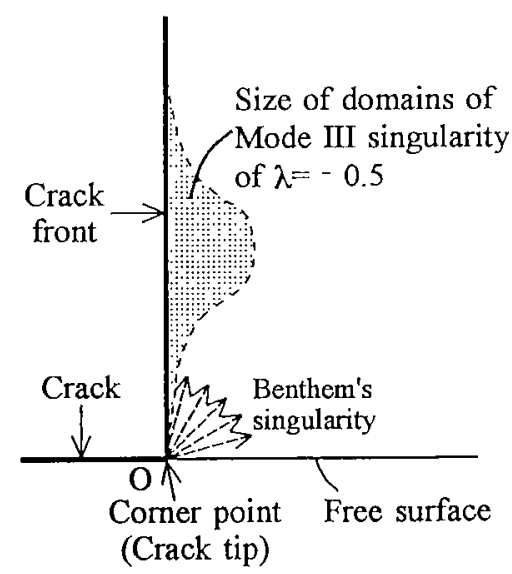

Fig. 22 Size of the domain having Mode III singularity of $\lambda=-0.5$

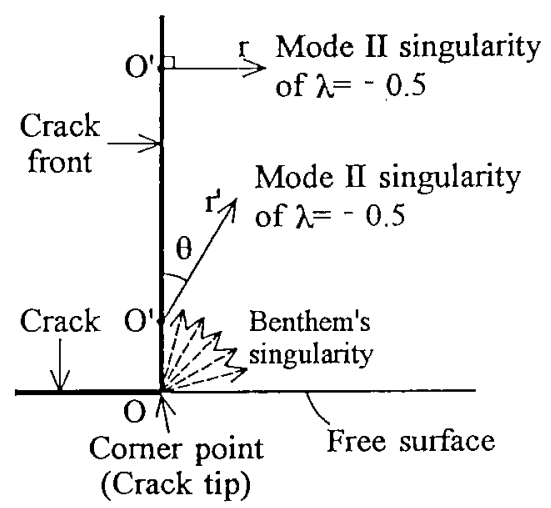

Fig. 23 Mode II singularity near the corner point

by Bazant et al., $F_{\text {II }}$ and $F_{\text {II }}$ approach constant values near the corner point.

\section{Schematic Illustration of Singular Stress Field near the Corner Point}

Considering the results obtained in this study and those of Benthem ${ }^{(1),(2)}$ and Bazant et al. ${ }^{(4)}$, the singular stress field for Mode III can be schematically illustrated as in Fig. 22. If we take a spherical coordinate system with the origin 0 at the corner point, we have the stress singularity pointed out by Benthem ${ }^{(1),(2)}$ in all direction in an infinitesimally small region near the corner point. On the other hand, if we put the origin of the polar coordinate system at a point $0^{\prime}$ along the internal crack front, the stress singularity in any direction at the crack tip becomes -0.5 .

Regarding Mode II singularity, the $\sqrt{r}$ singularity always holds in any direction except at the corner point as illustrated by Fig. 23.

\section{Conclusions}

The stress singularity for Mode II loading near the corner point where the crack front of a $3 \mathrm{D}$ surface crack intersects the free surface of a plate was analyzed by FEM models based on careful meshing. The existence of the Mode III stress intensity factor at the corner point reported by several papers contradicts with the boundary condition of the free surface, i.e., the shear stress related to $K_{\text {III }}$ should be zero at the corner point. In this paper, this paradox was solved by the detailed investigation of the singular stress field in the vicinity of the corner point. The conclusions can be summarized as follows.

(1) The stress intensity factors $K_{\mathrm{II}}$ and $K_{\mathrm{III}}$ along the crack front of a through crack and a semi-circular crack under Mode II loading increase steeply as the crack front approaches the corner point. However, the size of the domain of $K_{\text {III }}$ singularity shrinks toward zero as the crack front approaches the corner point. Thus, the paradox between the existence of $K_{I I I}$ and the boundary condition of the free surface has been solved.

(2) The finite values of $K_{\text {III }}$ at the corner point reported by existing studies contradict the boundary condition of free surface and are caused by the FEM calculation in which the coarse finite elements near the corner point cannot well model the steeply varying stresses.

(3) For the partially semi-circular crack whose front has a critical intersection angle of $67.0 \mathrm{deg}$. with the free surface, the stress singularity is -0.5 along the entire crack front and the values of $K_{\text {II }}$ and $K_{\text {III }}$ approach a constant value toward the corner point.

\section{References}

(1) Benthem, J.P., State of Stress at the Vertex of a Quarter-Infinite Crack in a Half-Space, Int. J. Solids Struct., Vol. 13 (1977), pp. 479-492.

(2) Benthem, J.P., The Quarter-Infinite Crack in a Half Space; Alternative and Additional Solutions, Int. J. Solids Struct., Vol. 16 (1980), pp. 119130.

(3) Bazant, Z.P., Three-Dimensional Harmonic Functions near Termination or Intersection of Gradient Singularity Lines: A General Numerical Method, Int. J. Eng. Sci., Vol. 12 (1974), pp. 221243.

(4) Bazant, Z.P. and Estenssoro, L.F., Surface Singularity and Crack Propagation, Int. J. Solids Struct., Vol. 15 (1979), pp. 405-426.

(5) Ghahremani, F., Numerical Variational Method for Extracting 3D Singularities, Int. J. Solids Struct., Vol. 27 (1991), pp. 1371-1386.

(6) Nakamura, T. and Parks. D.M., Three-Dimensional Elastic Stress Field near the Crack Front of a Thin Elastic, Plate, J. Appl. Mech., Vol. 55 (1988), pp. 805-813.

(7) Su, X.M. and Sun, C.T., On Singular Stress at the Crack Tip of a Thick Plate under In-Plane Load- 
ing, Int. J. Fract., Vol. 82 (1996), pp. 237-252.

(8) Tohgo, K., Otsuka, A. and Yuuki, R., Fatigue Crack Growth of a Mixed Mode Three-Dimensional Crack (1st Report, Analysis of Stress Intensity Factors of Mixed Mode Three-Dimensional Cracks Based on the J-Integral Concept)., Trans. Jpn. Soc. Mech. Eng., (in Japanese), Vol. 52, No. 476, A (1986), pp. 909-918.

(9) Otsuka, A., Tohgo, K. and Yoshida, M., Fatigue Crack Growth of a Mixed Mode Three-Dimensional Crack (2nd Report, Fatigue Crack Growth Behavior from a Semi-Elliptical Surface Crack under Shear Loading), Trans. Jpn. Soc. Mech. Eng., (in Japanese), Vol.54, No. 505, A (1988), pp. 1735-1744, See also Stress Intensity Factors Handbook, Ed-in-chief, Murakami, Y., Vol. 3 (1992), pp. 713-714, Soc. Materials Sci., Japan, Kyoto \& Pergamon Press, Oxford.

(10) Rice, J.R., A Path-Independent Integral and the Approximate Analysis of Strain Concentration by Notches and Cracks, J. Appl. Mech., Vol. 35 (1968), pp. 379-386.

(11) He, M.Y. and Hutchinson, J.W., Surface Crack
Subject to Mixed Mode Loading, Eng. Fract. Mech., Vol. 65 (2000), pp. 1-14.

(12) Pook, L.P., A Note on Corner Point Singularities, Int. J. Fract., Vol. 53 (1992), pp. R3-R8.

(13) Pook, L.P., Some Implications of Corner Point Singularities, Eng. Fract. Mech., Vol. 48 (1994), pp. 367-378.

(14) Pook, L.P., Crack Profiles and Corner Point Singularities, Fatigue Fract. Engng. Mater. Struct., Vol. 23 (2000), pp. 141-150.

(15) Pook, L.P., Finite Element Analysis of Corner Point Displacements and Stress Intensity Factors for Narrow Notches in Square Sheets and Plates, Fatigue Fract. Engng, Mater. Struct., Vol. 23 (2000), pp. 979-992.

(16) Dhondt, G., On Corner Point Singularitis along a Quarter Circular Crack Subject to Shear Loading, Int. J. Fract., Vol. 89 (1998), pp. L33-L38.

(17) Kassir, M.K. and Sih, G.C., Three-Dimensional Stress Distribution around an Elliptical Crack under Arbitrary Loadings, J. Appl. Mech., Vol. 33 (1966), pp. 601-611. 